epithels und ist ebenso wie die Basalmembran und subepitheliale Schicht reichlich mit Rundzellen durchsetzt. Im Stroma der Schleimbaut zahlreiche degenerirte Drüsenschläuche. Das Periost verdickt, der Knochen verdünnt. Bei a eine $\mathrm{Ha}$ w ship'sche Lacune mit Osteoblast. Bei c eine sog. Mastzelle. Bei b Markcanälchen mit Blutgefäss. Homog. Immersion. $\frac{1}{12}$ Leitz. Ocul. Hartnak N. 3.

\title{
III.
}

\section{Zur Histo-Pathologie des Pankreas beim Diabetes mellitus.}

(Aus dem Pathologisehen Laboratorium der Chicagoer Poliklinik.) Von

Dr. Maximilian Herzog,

Professor der Pathologie an der Chicagoer Poliklinik, Chicago, Ill. U. S. A. (Hierzu Taf. III.)

Die Frage, ob sich beim Diabetes mellitus im Pankreas specifische Veränderungen vorfinden, ist in allerletzter Zeit von verschiedenen Seiten zum Gegenstand eingehender Untersuchungen gemacht worden. Da auch ich, seit Erscheinen der vorläufigen Mittheilung von Ssobolew und des ersten Berichtes von Opie, in Verbindung mit anderen Arbeiten über dies Organ, die Bauchspeicheldrüse in mehreren Fällen von Diabetes untersucht habe, und da soeben eine auf breiter Basis angelegte bedeutsame Mittheilung von Weichselbaum und Stangel erschienen ist, so möchte ich einen kurzen Bericht meiner eigenen, noch nicht abgeschlossenen Beobachtungen geben.

Der Gedanke, beim Diabetes mellitus auf typische Veränderungen im Pankreas zu fahnden, ist keineswegs neu, er entstand direct im Anschluss an die von Mering'schen und Minkowski'schen Versuche, welche zeigten, dass totale Pankreasexstirpation bei gewissen Thieren, bei denen sich diese Operation überhaupt durchführen lässt, zum tödtlich verlaufenden Diabetes 

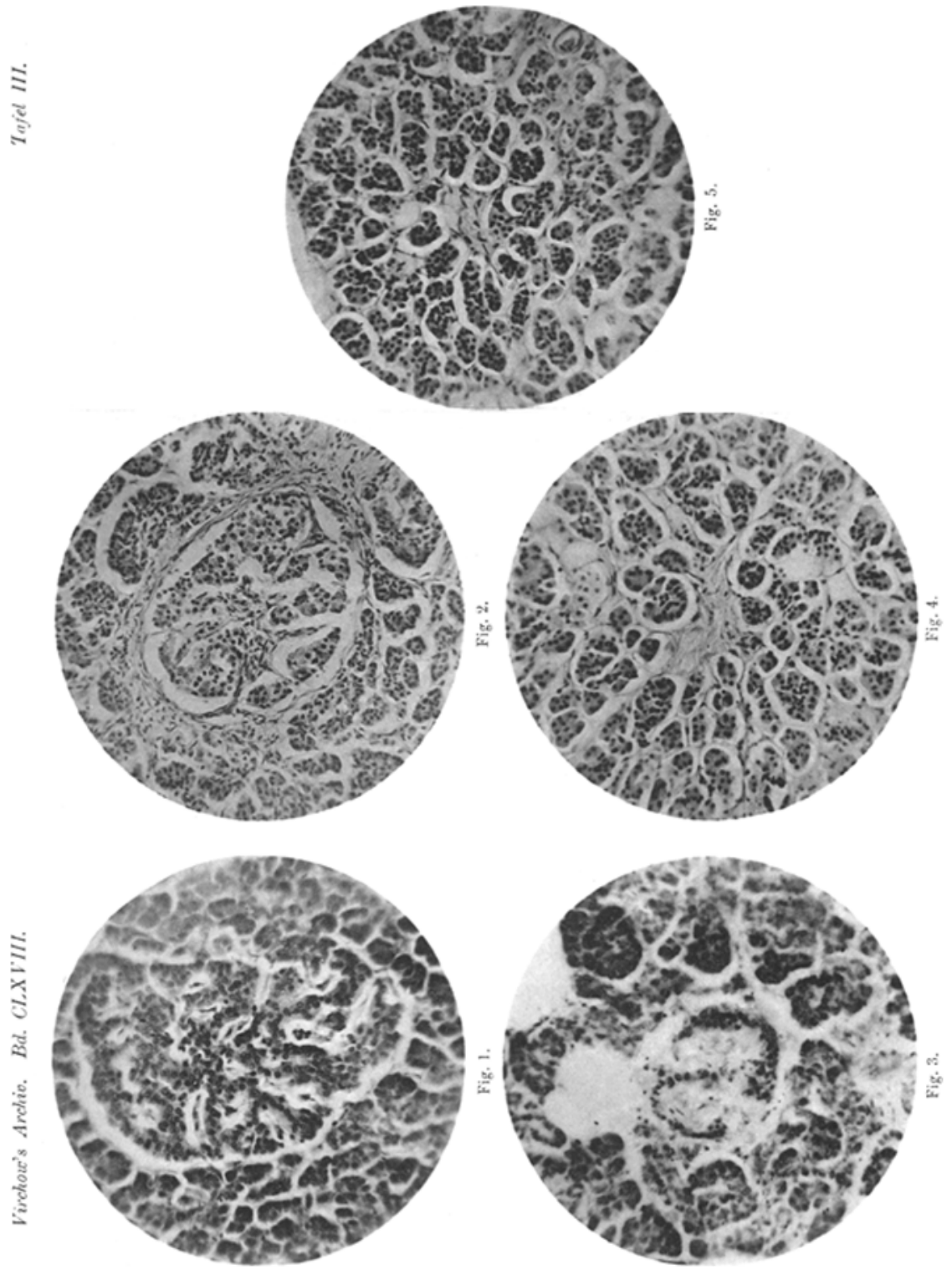
führt. Diese Thatsache demonstrirte, dass das Pankreas bezüglich der Umsetzung der Kohlehydrate im Thierkörper gewisse essentielle Functionen ausübt, welche, nach Entfernung der Bauchspeicheldrüse, von keinem anderen Organ übernommen werden können. Histologische Veränderungen im Pankreas, wie sie zuerst beschrieben wurden, boten indessen nichts Specifisches, da sie einerseits weder constant vorhanden waren, andererseits auch bei Pankreas-Affectionen ohne Diabetes gefunden wurden. Gewisse eigenartige Gebilde im Pankreas, rundliche oder unregelmässige Haufen von Zellen, verschieden von den gewöhnlichen secernirenden Parenchymzellen, die bekannten Langerhans'schen Inseln, hatten schon vor Längerem die Aufmerksamkeit der Untersucher erregt. Es war festgestellt worden, dass die innerhalb der Acini belegenen Langerhans'chen Zellhaufen keine Ausführungsgänge haben, und nachdem Kuehne und Lea dann die weiten Blutcapillaren der Inseln beschrieben hatten, bildete sich die Ansicht aus, dass die letzteren eine sogenannte innere Secretion liefero. Von Laguesse, Schaefer, Dimare und Anderen war dann später der Gedanke ausgesprochen worden, dass die Langerhans'schen Inseln zu der Umsetzung der Kohlehydrate im Thierkörper in causalem Zusammenhange stehen möchten.

Auf specifische Veränderungen dieser Inseln beim Diabetes mellitus ist nun in letzter Zeit hingewiesen worden. Die erste Arbeit, in der eine solche Angabe mit Nachdruck gemacht wird, ist die von Ssobolew ${ }^{1}$ ), der in einer vorläufigen Mittheilung angiebt, dass er in zwei Fällen von Diabetes mellitus in den untersuchten Bauchspeicheldrüsen überhaupt keine Langerhans'schen Inseln mehr vorfand; Ssobolew giebt ferner an, dass die Unterbindung des Ductus Wirsungianus, welche zu Atrophie des Pankreas ohne Diabetes führt, die Langerhansschen Inseln nicht afficirt, so dass das verbleibende Gewebe geradeza überhaupt nur aus Langerhans'schen Inseln besteht. $0 \mathrm{pi}^{2}$ ) hat mehrere Arbeiten über Pankreas-Veränderungen bei Diabetes veröffentlicht und giebt an, dass seine Untersuchungen

1) Ssobolew, Centralblatı für Patbologie, 1900, Bd. 11, S. 102 .

$\left.{ }^{2}\right)$ Opie, Journ. of Experim. Medic., 1900, Vol. 5, No. 4 and No. 5, and Journal of the Boston Soc. of Med. Science, 1900, Vol. 4. 
bereits im Gange waren, als die Ssobolew'sche vorläufige Mittheilung erschien. Opie unterscheidet zwei Arten von interstitiellen Pankreas-Veränderungen, eine interlobuläre und eine interacinöse Bindegewebs-Wucherung. Bei der letzteren bemerkte er auch eine Verdickung des die Inseln umgebenden Bindegewebes und eine Wucherung des feinen Bindegewebes der InselCapillaren. Die Zellen der Inseln beschreibt 0 pi e als verkleinert, die Kerne als gleichfalls klein und sich tief färbend. Im Ganzen hat Opie 11 Fälle von Diabetes untersucht, und in 4 Fällen fand er so prägnante Veränderungen der Langerhans'schen Inseln, dass er glaubt, denselben genetische Beziehungen zum Diabetes zugestehen dürfen zu können. Bei einem 17jährigen Mädcben, das seit 2 Jahren an Diabetes gelitten hatte, fánden sich im Pankreas und zwar am zahlreichsten im lienalen Ende, das nach 0 pie am reichsten an Inseln ist, hyaline Heerde, deren grösste beinahe $1 \mathrm{~mm}$ im Durchmesser hatten, so dass man sie mit unbewaffnetem Auge sehen k'onnte. Die Heerde bestanden aus homogener, hyaliner Substanz, und sie fanden sich zwischen wenig oder nicht veränderten Acini, die Inseln dagegen waren verschwunden, es schien die hyaline Substanz an ihre Stelle getreten zu sein. In einem anderen Falle, den einer 54jährigen Negerin, beschreibt 0 pie die folgenden Veränderungen der Inseln. Eine allgemeine interstitielle Bindegewebs-Neubildung fehlte, dagegen fanden sich hier und da, besonders im Schwanzende, einige wenige Bindegewebs-Wucherungen. In beinabe jeder Insel fand sich mehr oder weniger homogenes Material, das sich mit Eosin färbte. Nur selten sah man eine unveränderte Insel; die am wenigsten veränderten zeigen einige zerstreute hyaline Schollen. Diese letzteren waren unregelmässig polygonal und entsprachen an Grösse den Inselzellen. Zuweilen nahm das hyaline Material fast die ganze Insel ein. Opie glaubt, dass das Hyalin wahrscheinlich epithelialen Ursprunges ist. Eiue sehr bedeutsame Arbeit über Pankreas-Veränderungen beim Diabetes mellitus haben vor Kurzem Weichselbaum und Stang $\mathrm{l}^{1}$ ) veröffentlicht. Die Untersuchung der beiden Autoren stätzt sich auf das Material von 18 Fällen von Diabetes und

1) Weichselbaum und Stangel, Wiener klin. Wochensehrift, 1901, No. 41, S. 968 . 
sie finden eine Atrophie, welche speciell die Langerhans'schen Inseln ergreift, während andererseits bei allen anderen von den Genannten untersuchten Pankreas-Affectionen ohne Diabetes die Inseln ausserordentlich gut erhalten sind.

Nachdem ich bereits über meine eigenen Pankreas-Untersuchungen bei Diabetes mellitus einen Vortrag in der Chicagoer Pathologischen Geselischaft gehalten hatte, erschien noch eine Arbeit von Wright und Joslin ${ }^{1}$ ), Dieselben haben PankreasGewebe in 9 Fällen von Diabetes untersucht. Es standen ihnen jedoch in fast allen Fällen nur 1-3 Schnitte zur Verfügung, so dass den Ergebnissen dieser Untersuchung nicht allzu viel Werth beizulegen ist. Wright und Joslin fanden in zwei von den 9 untersuchten Fällen hyaline Veränderungen der Langerhans'schen Inseln, so wie sie vorher 0 pie beschrieben hatte.

Das Material, das ich selbst untersucht habe, entstammt 5 Fällen von Diabetes mellitus. In einem Falle nahm ich selbst die Section vor und lernte die Details der Anamnese des Falles kennen. Für die übrigen 4 Fälle bin ich Herrn Collegen Ludwig Hektoen zu grossem Danke verpflichtet. Von 3 dieser 4 Fälle erhielt ich nur kleine Gewebsstïckchen; dieselben kamen von Museums-Präparaten, von denen weiter Nichts bekannt war, als dass sie Fällen von Diabetes mellitus entstammten. Die 3 Drüsen waren klein und mehr oder weniger unter dem Durchschnittsgewicht. Der vierte Fall kam in letzter Zeit zur Section. Genau untersucht werden. konnten im Ganzen 3 Fälle, in 2 war das Material einer eingehenden Untersuchung nicht günstig. Es liessen sich indessen für alle 5 Fälle unverkennbar Veränderungen an den Inseln feststellen. Etwas, was bei allen fünfen auffiel, und was besonders Weichselbaum und Stangel hervorgehoben haben, ist die numerische Verminderung der Inseln, die von einer geringen Verminderung in Fall 1 bis zum totalen Schwund in Fall 5 variirte. Ich habe zuerst die Angabe Ssobolew's, dass er bei seinen beiden Fällen von Diabetes mellitus überhaupt keine Inseln mehr vorfand, stark bezweifelt, aber meine Meinung geändert, als es mir unmöglich war, im eigenen Fall 5 Inseln zu finden, dieselben sind thatsächlich total verschwunden, und

1) Wright und Joslin, Journal Med. Research, Vol. 4, 1901, November p. 360 . 
Alles, was an sie erinnert, sind spärliche Bindegewebsknötchen, die sich hier und da an Stellen finden, die früher von Inseln eingenommen worden sind.

Die mikroskopiscbe Untersuchung des Materials wurde an Paraffinschnitten, die in Haematoxylin und Erythrosin und Eosin und alkalischem Methylenblau gefärbt worden waren, vorgenommen. Sie hatte die folgenden Ergebnisse:

Fall 1. Das Pankreas stammt von einem 40jährigen Kaufmann. Etwa ein Jahr, ehe der Patient starb, entdeckte man Zucker in seinem Harn. Die Zuckermenge war während des ganzen Krankheitsverlaufes nur eine mässige. Ein Jahr, nachdem der Diabetes zuerst constatirt worden war, stellte sich Coma diabeticum ein. Im Harn fanden sich jetzt neben Zucker noch Aceton und $\beta$-0xybuttersäure. Der Patient starb in Verlaufe des Coma-Anfalles. Bei der Section wurden alle inneren Organe als normal befunden mit folgenden Ausnahmen: Im Oberlappen der linken Lunge fand sich eine centrale Pneumonie, auch wareu linkerseits alte fibröse Adhäsionen zwischen Pleura pulmonalis und costalis vorbanden. Das Pankreas war an Länge normal, aber an Breite und Dicke stark vermindert, das Gewebe fühlte sich etwas härter als gewöhnlich an, trotzdem es eine măssige Menge Fett enthielt. Die Läppehen erscheinen auf der Schnittfläche schārfer abgegrenzt, als in der Norm. Ductus offen; keine Steine in demselben oder in seinen Verzweigungen.

Die mikroskopische Untersuchung der inneren Organe ergab nichts Pathologisches, bis auf die pneumonische Verdichtung und beginnende leichte parenchymatöse Veränderungen in den Nieren. Im Pankreas sind die folgenden Veränderungen za constatiren. Das intralobuläre Bindegewebe ist an vielen Orten vermehrt. Wenn auch die Vermehrung im Grossen und Ganzen sich in mässigen Grenzen hält, so ist dieselbe doch stellenweise recht bedeutend, so dass ziemlich breite Bindegewebs-Septen vorhanden sind. Dieselben schliessen dann stark verkleinerte Drüsenläppeben ein. Hier und da findet sich offenes Fettgewebe in kleineren Portionen vor. Die Venen in diesem offenen Gewebe sind vielfach von dichten Rundzellenheerden umgeben. Die infiltrirenden Zellen zeigen den Leukocyten-Typus. Die Langerhans'schen Inseln scheinen, verglichen mit normalem PankreasGewebe, an Zahl leicht vermindert; sie sind von stark verdickten Kapseln umgeben. Einzelne Irseln haben die Durchschnittsgrösse; andere sind mehr oder weniger verkleinert. In den letzteren sind die Epithelzellen klein und dicht zusammengepfercht. Andere Zellen haben ein undeutliches, wie aufgelöst oder angefressen aussehendes Protoplasma. In vielen Inseln sieht man, eine Vermehrung des innerhalb der Insel belegenen Bindegewebes, das dann die Capillare umgiebt, beziehentlich zwischen denselben liegt. Die Veränderungen des Inselgewebes, abgesehen von der sehr markanten Kapsel-Verdickung, sind indessen zumeist leichterer Art, Man darf wobl 
annehmen, dass in unserem Falle 1 jene wahrscheinlich für Diabetes charakteristischen Pankreas-Veränderungen noch nicht sehr stark entwickelt waren, da ja die Krankheit erst verhältnissmässig kurze Zeit und in milderer Form bestanden hatte. Das tödtlich verlaufende Coma diabeticum war vielleicht im Anschluss an die Pneumonie, die bei der Section constatirt wurde, entstanden.

Fall 2. Die Bindegewebs-Neubildung in diesem Falle ist bedeutend stärker, als in Fall 1. Besonders ausgeprägt ist die interacinöse Bindegewebs-Wucherung, allein auch das interlobuläre Bindegewebe ist vermehrt. Ganz bedeutend ist die Verfettung, und an einzelnen Stellen sind ganze Gruppen von Läppchen durch offenbares Fettgewebe ersetzt. Es finden sich nur wenige Inseln, Keine derselben erscheint normal, es bestebt KapselVerdickung, Vermebrung des intra-insularen Bindegewebes. Die Schnitte von Fall 2 färbten sich nichts weniger als befriedigend.

Fall 3. Das interacinöse Bindegewebe ist sehr mässig vermehrt, das interlobuläre gleichfalls. Verfettung recht mässig. Anfangs schien es, als ob sich einzelne normale oder fast normale Inseln vorfänden, aber bei wiederholter genauerer Untersucbung fanden sich doch an allen Inseln Veränderungen gleicher Art, aber dem Grade nach stark variirend. Die grosse Nebrzahl der Inseln zeigt tiefgehende Veränderungen. Es findet sich keine Kapsel-Verdickung, dagegen sind im Centrum der Inseln die Epithelzellen grösstentheils verschwunden, ihre Stelle wird von einem mehr oder weniger structurlosen Material eingenommen. Dasselbe bestebt aus unregelmässigen Schollen, die zuweilen wie geschwollene, degenerirte Zellen ausseben. Zuweilen sehen die hyalinen Massen auch so aus, dass man an die Möglichkeit denken kann, dass sie von hyalin degenerirten Capillaren abstammen. Die Schnitte in Fall 3 färbten sich ziemlich gut, doch gaben sie"keinen unzweifelhaften Aufschluss über die Abstammung der byalinen Substanz in den Inseln.

Fall 4. Das interacinöse Bindegewebe ist mässig vermehrt, das interlobuläre stellenweise ziemlich beträchtlich. Es sind fast gar keine Inseln vorhanden. Erst nach vieler Hühe fanden sich ein paar, dieselben sind klein, haben eine etwas verdickte Kapsel, zeigen dagegen weder hyalines Material, noch intra-insuläre Bindegewebs-Wucherung. Es wurden in diesem Falle nur eine mässige Anzahl Schnitte untersucht. Dieselben färbten sich nicht sehr gut.

Fall 5. Das Pankreas entstammt einem 30jährigen Manne, der an einem Diabetes schwerster Form mit grossen Zuckermengen im Harn gelitten hatte. Er war, als er zur Section kam, stark abgemagert. Es fand sich eine suppurative Pleuritis der rechten Seite mit Abscess und Gangrän im rechten Unterlappen. Das Pankreas war von mässiger Grösse, von normaler Consistenz mit sehr mässiger Fett-Infiltration. Gewicht 90-100 gr. Es wurden von dieser Bauchspeicheldrüse mehrere hundert Schnitte, 
darunter Serien von 50 Schnitten von $20 \mu$ Dicke, natürlich auch ganz feine Schnitte, untersucht. Das interlobuläre und das interacinöse Bindegewebe sind beide sehr mässig vermehrt. Fettgewebe nur vereinzelt in kleinen Fleckehen vorhanden. Auch nichtin einem einzigen Schnitte konnte eine Langerhans'sche Insel aufgefunden werden. Hier und da schien es, als ob eine ganz kleine Insel mit wenigen comprimirten Zellen vorbanden wäre, aber wiederholte Untersuchung führte zu der Ueberzeugung, dass es sich hier nicbt um Inselgewebe, sondern um Gruppen. von centro-acinären Zellen handle. Dagegen fanden sich in fast allen Schnitten im Centrum oder nahe der Peripherie der Läppchen, d. h. also an Stellen, wo man normaler Weise Inseln findet, kleine, rundliche oder unregelmässig polygonale Massen von Bindegewebe. Diese Knötchen zeigen eine mässige Zahl von Spindelzellen, eingebettet in feinerer oder gröberer fibrillärer Matrix. Diese intralobulären Bindegewebs-Knötchen fanden sich im Schwanz-Ende zahlreicher, als irgendwo anders. Es scheint ganz entschieden, als ob diese Bindegewebs-Knötchen die Ueberreste früherer Langerhans'scher Inseln seien.

$\mathrm{Zu}$ dem obigen Bericht ist $\mathrm{zu}$ bemerken, dass die Untersuchung Ergebnisse geliefert hat, welche sowohl die Angaben von Ssobolew, Opie, als auch die von Weichselbaum und Stangel bestätigen. Die Letzteren melden in ihrer Arbeit Nichts von hyaliner Degeneration der Inseln, wie sie Opie in zweien seiner Fälle und wir in einem der unserigen saben. Ssobolew giebt an, dass er in den Bauchspeicheldrüsen von 2 Fällen von Diabetes überhaupt die Inseln ganz vermisste, und in unserem Fall 5, in dem das Gewebe zumeist sehr gut in Zenker'scher Lösung sich conservirt hatte und die Schnitte sich sehr schön färbten, gelang es uns gleichfalls nicht, Inseln zu finden. Bezüglich der Deutung der vorgefundenen pathologischen Veränderungen möchten wir uns Weichselbaum und Stangel anschliessen. Dieselben fassen den Vorgang als eine specifische Atrophie des Inselgewebes aus unbekannten Ursachen auf.

Es scheint nun, als ob diese Atrophie nicht stets in der gleichen Weise vor sich geht. Es kann zur Bildung von hyalinem Material an Stelle der Inseln kommen, oder es könnon die Inselzellen unter gleichzeitiger Bindegewebs-Wucherung einfach schwinden, ohne dass sich hyalines Material vorfindet. Geradezu verblüffend wirkt es, wenn man, wie in unserem Fall 5 , in Hunderten von Schnitten überhaupt keine Inseln mehr vorfindet und als einzige mögliche Spur derselben kleine Bindegewebs- 
Knötchen antrifft. Im Fall 5, einem sehr schweren DiabetesFalle, waren alle anderen Veränderungen im Pankreas-Gewebe, abgesehen von dem Inselschwunde, ganz unbedeutender Natur.

Die neueren Untersuchungen über die Histiologie des Pankreas beim Diabetes scheinen entschieden darauf hinzuweisen, dass bei dieser geheimnissvollen Erkrankung die Langerhansschen Inseln in specifischer Weise betroffen sind. Diese allem Anscheine nach specifischen Veränderungen sprechen nun ihrerseits zu Gunsten der Ansicht, welche den Langerhans'schen Inseln die innere Secretion eines Zucker umsetzenden Enzyms zuschreibt.

\section{Erklärung der Abbildungen auf Tafel III.}

Die Mikro-Photographien sind alle bei derselben Vergrösserung angefertigt, nehmlich Spencer's Objectiv (professional) t Zoll; Ocular 1 Zoll.

Fig. 1. Schnitt aus dem normalen Pankreas eines an Hüftgelenk:Tuberculose zu Grunde gegangenen Kindes, das ganz kurze Zeit nach Eintritt des Exitus secirt wurde. Stückchen der Bauchspeicheldrüse wurden sofort nach Eröffnung der Leibesböhle in Zenkersche Läsung gebracht. In der Mitte normale Langerhans'sche Insel.

Fig. 2. Schnitt aus dem Pankreas von Diabetes-Fall 1. Die Kapsel der Langerbans'schen Insel verdickt; Entwicklung von intra-insulärem Bindegewebe. Degenerirte Epithelzellen ohne Kern.

Fig. 3. Schnitt aus dem Pankreas in Diabetes-Fall 3. In der Mitte kleine Insel mit hyaliner Substanz im Centrum. In der Nähe der Insel verfettete Partien.

Fig. 4 u. 5. Schnitte aus dem Pankreas von Fall 5. Im Centrum des Gesichtsfeldes Bindegewebs-Knötchen, die Ueberreste von Langerhans'schen Inseln. 